\title{
Religião e fecundidade entre adolescentes no Brasil
}

\author{
Ana Paula de Andrade Verona1 e Cláudio Santiago Dias Júnior ${ }^{2}$
}

Como citar Verona APA, Dias Júnior CS. Religião e fecundidade entre adolescentes no Brasil. Rev Panam Salud Publica. 2012;31(1):25-31.

RESUMO Objetivo. O objetivo deste estudo foi examinar a associação entre a idade ao ter o primeiro filho durante a adolescência e antes do casamento e o envolvimento religioso no Brasil, medido através da afiliação religiosa e frequência aos cultos ou missas.

Métodos. Foram utilizados dados transversais e obtidos na Pesquisa Nacional de Demografia e Saúde de 1996 e da Pesquisa Nacional de Demografia e Saúde de Mulheres e Crianças de 2006. Foram empregados modelos de riscos proporcionais de Cox para estimar a associação entre religião e idade ao ter o primeiro filho pré-marital e durante a adolescência.

Resultados. Os resultados indicam uma forte associação entre fecundidade pré-marital na adolescência e envolvimento religioso, tanto em 1996 como em 2006. Em 1996, a frequência aos cultos ou missas foi mais importante na explicação da idade ao primeiro filho do que a afiliação religiosa. Em 2006, o pertencimento a uma igreja pentecostal passa a ser predominante.

Conclusões. Os resultados apresentados neste trabalho são instigantes, na medida em que evidenciam que adolescentes protestantes, particularmente pentecostais, apresentam um risco reduzido de serem mães adolescentes e antes do casamento. Este resultado não era esperado, já que o pentecostalismo predomina nos grupos populacionais menos favorecidos, com menor renda, educação e residentes nas áreas urbanas, onde também se concentra a fecundidade de adolescentes no Brasil. Futuros estudos devem ser conduzidos a fim de compreender como os distintos mecanismos da influência religiosa atuam na vida e comportamento de adolescentes no Brasil.

Palavras-chave Religião e sexo; adolescente; comportamento sexual; comportamento reprodutivo; Brasil.

No Brasil, ao contrário da maioria dos países em desenvolvimento, tem sido observado um aumento considerável na proporção de adolescentes que já iniciaram a vida sexual $(1,2)$. Segundo a Pesquisa Nacional de Demografia e Saúde realizada no Brasil em 1996 (PNDS 1996) (3), 33\% das mulheres entre 15 e 19 anos responderam que já tinham tido uma relação sexual, enquanto dados da Pes-

\footnotetext{
Universidade Federal de Minas Gerais, Departamento de Demografia/Cedeplar, Belo Horizonte (MG), Brasil. Enviar correspondência a: Ana Paula de Andrade Verona, anapaulaverona@gmail.com

2 Universidade Federal de Minas Gerais, Departamento de Sociologia e Antropologia, Belo Horizonte (MG), Brasil.
}

quisa Nacional de Demografia e Saúde da Criança e da Mulher de 2006 (PNDS 2006) (4) mostraram que esta proporção tinha aumentado para 55\%. Este cenário ajuda a explicar o comportamento da fecundidade entre as adolescentes no Brasil, que aumentou nos anos $80 \mathrm{e}$ 90 (5), e recentemente tem se mantido estável. Mesmo assim, a contribuição proporcional deste grupo para a taxa de fecundidade total (TFT) continuou crescendo, passando de 17\% em 1996 para 23\% em 2006. Ou seja, em 2006, aproximadamente um em cada quatro nascimentos no Brasil ocorreu entre adolescentes entre 15 e 19 anos, o que sugere um problema de saúde pública. Esta proporção é muito superior à mundial (6) e ao que seria esperado para o Brasil, já que o nível educacional e o acesso aos métodos contraceptivos também têm crescido no país (7).

Evidências empíricas recentes têm sugerido que a religião pode ser um fator associado ao comportamento sexual e reprodutivo de adolescentes no Brasil (8-11). Distintos mecanismos têm sido teorizados na tentativa de explicar como esta associação acontece (12). Um dos mais considerados é o papel das normas e ensinamentos religiosos sobre o que é certo ou errado, aceitável ou 
não, dentro de cada religião ou igreja. Algumas normas, como a oposição ao sexo pré ou extra-marital pode estar associada a um menor risco de iniciar a vida sexual precocemente, ou mesmo de ter múltiplos parceiros sexuais, reduzindo assim, o risco de ser mãe na adolescência ou de contrair doenças sexualmente transmissíveis. Além desse, outros mecanismos de influência religiosa podem ser observados quando a religião oferece um espaço de socialização, onde adolescentes têm a oportunidade de aprender diferentes técnicas, como de liderança e de superação de momentos difíceis (13). Outros mecanismos ainda salientam o importante papel das interações sociais, em especial com adultos e líderes religiosos, que podem servir como valiosas fontes de informação, cuidado e supervisão para os adolescentes. A obtenção destas técnicas e $o$ acesso a tais interações podem ser utilizadas em outras esferas da vida do adolescente, como na escola ou no mercado de trabalho, o que pode indiretamente (e positivamente) influenciar o seu comportamento sexual e reprodutivo (13-16).

Com o recente crescimento do protestantismo no Brasil, a disseminação de normas e padrões do comportamento sexual e reprodutivo de adolescentes e jovens solteiros passou a ter papel de destaque no campo religioso brasileiro, uma vez que os protestantes, especialmente os pentecostais, abordam explicitamente este tema. Burdick (14), por exemplo, observou em seu trabalho de campo, que o sexo antes do casamento é considerado um pecado grave para membros da igreja Assembléia de Deus, e que aqueles que desobedecem esta norma podem ser punidos através da disciplina - uma exclusão temporária de atividades que participam na igreja. Mariz (15) relatou resultados similares no que tange a gravidez antes do casamento entre membros de igrejas pentecostais.

Além disso, as igrejas pentecostais têm oferecido cada vez mais espaço para a atuação e envolvimento de adolescentes e jovens em um contexto religioso, mantendo assim uma relação bastante próxima com este grupo. Cultos de jovens e de namorados, viagens, cursos bíblicos e profissionalizantes, palestras sobre comportamento e saúde, mobilizações na ajuda de comunidades carentes, entre outras atividades, como auxílio na busca por emprego e incentivo ao estudo, têm sido frequentemente observadas em igrejas protestantes pentecostais (16).
Tais recursos podem contribuir para a criação de mecanismos através dos quais a religião pode influenciar, mesmo que indiretamente, a vida e o comportamento de adolescentes e jovens no Brasil.

Considerando as recentes transformações no campo religioso brasileiro, em particular, o aumento do pentecostalismo, e a potencial influência da religião no comportamento e estilo de vida dos adolescentes, o objetivo deste estudo foi examinar a associação entre filiação religiosa e frequência aos cultos ou missas e a idade ao ter o primeiro filho prémarital durante a adolescência.

\section{MATERIAIS E MÉTODOS}

Este estudo utilizou dados de natureza transversal com representatividade nacional retirados das PNDS 1996 e 2006. Estas pesquisas foram realizadas sob o marco do Programa Demographic and Health Survey (DHS), pela Sociedade Civil Bem-Estar Familiar no Brasil (BEMFAM) e pelo Centro Brasileiro de Análise e Planejamento (CEBRAP), respectivamente. As PNDSs são amostras probabilísticas complexas estratificadas em dois estágios: unidades primárias compostas por setores censitários, e unidades secundárias, formadas pelas unidades domiciliares, sendo necessário especificar seus respectivos pesos e unidades amostrais. Para 1996 as informações necessárias para esta especificação estão disponíveis nas variáveis de estrato (sete regiões) e de conglomerado (842 setores). E para 2006 as unidades amostrais levam as mesmas denominações (sendo o estrato composto por 10 unidades e os conglomerados por 1088 setores). No caso dos pesos amostrais, para 1996 utilizou-se o fator de ponderação do grupo analisado (peso da mulher elegível dentro do domicílio) e para 2006, o fator de expansão do grupo correspondente (17).

É importante salientar que ao se especificar os pesos e unidades amostrais das PNDSs de 1996 e 2006, as mesmas se tornam totalmente comparáveis (17). No software STATA, versão 10, esta especificação pode ser feita através do comando "svyset", que produz estimativas de erro padrão e de testes estatísticos corrigidas pela complexidade da amostra. Toda análise descritiva e estatística apresentada neste trabalho foi corrigida por este comando.

Sabendo que a história de nascimentos de adolescentes é incompleta, a expe- riência de jovens adultas (entre 20 e 24 anos, e que recentemente deixaram a adolescência) também é considerada. A amostra final totaliza 4528 mulheres entre 15 e 24 anos em 1996 e 4996 em 2006. Além disso, devido aos efeitos de confundimento entre casamento e início da vida sexual e reprodutiva, este trabalho examina o risco de ter o primeiro filho antes do casamento - ou seja, quando a idade da mulher ao ter o primeiro filho é menor que a idade à primeira união - seja essa formal ou não - ou quando a mãe nunca esteve em uma união. As duas PNDSs medem tais idades em anos completos, gerando assim estimativas conservadoras sobre o comportamento pré-marital. Isto porque alguns dos nascimentos podem ter ocorrido poucos meses antes ou depois do casamento, mas na mesma idade completa, sendo contados, então, como eventos maritais (18).

A PNDS de 1996 classifica a filiação religiosa em oito categorias: Católico, Protestante, Evangélico, Espírita, religiões Afro-Brasileiras; religiões orientais, Judeus, e outras religiões. A PNDS de 2006, por outro lado, classifica a filiação religiosa em seis grupos: Católico, Protestante tradicional, Pentecostal, Espírita, religiões Afro-Brasileiras, e outras religiões. Para garantir a comparabilidade entre as duas pesquisas foi necessário re-categorizar a variável de filiação religiosa, que foi agrupada da seguinte maneira: Catolicismo (grupo de referência), Protestantismo (que inclui os protestantes e evangélicos em 1996, e os protestantes tradicionais e pentecostais em 2006). Os demais grupos de religião foram reagrupados em uma única categoria chamada de Outras religiões. Por fim, a variável de religião também inclui uma categoria para os que reportaram não ter uma filiação religiosa.

Já a variável de frequência aos cultos ou missas foi classificada em cinco grupos tanto em 1996 como em 2006. Novamente, para garantir a comparabilidade entre estes anos, a variável referente à frequência aos cultos ou missas foi reagrupada em quatro grupos, sendo estes: frequenta a cultos ou missas uma vez por semana ou mais ( 1 ou + vzs/ semana), menos de uma vez por semana (- $1 \mathrm{vz} /$ semana), menos de uma vez por mês (- $1 \mathrm{vz} / \mathrm{mês})$, nunca (grupo de referência).

Este estudo emprega modelos de riscos proporcionais de Cox (19), o qual além de permitir a análise de dados 
com uma dimensão temporal, também possibilita a incorporação de diferentes covariáveis que poderiam confundir a associação entre religião e fecundidade. Os resultados são apresentados em três modelos para cada ano de análise, $1996 \mathrm{e}$ 2006. Os primeiros dois modelos examinam se a razão de risco de uma mulher ter um filho durante a adolescência e antes do casamento está associada com a filiação religiosa e a frequência aos cultos ou missas.

O terceiro modelo apresenta a associação entre as razões de riscos e variáveis que representam combinações entre filiação religiosa e frequência aos cultos ou missas. Tais variáveis foram criadas para que estas duas informações de religião pudessem ser usadas simultaneamente, sem a ocorrência de multicolinearidade decorrente da alta correlação entre elas (como sugerido na Tabela 2). Foram então criadas seis variáveis dicotômicas mutuamente excludentes que representam combinações entre as filiações religiosas e duas categorias de frequência aos cultos ou missas, que são mulheres que atendem aos cultos ou missas com alta frequência ( 1 ou + vzs/semana) e mulheres que frequentam os cultos ou missas com baixa frequência $(-1 \mathrm{vz} /$ semana). As seis variáveis foram assim construídas: católicas que frequentam a missa com alta frequência (católico, 1 ou + vzs/semana), católicas que frequentam a missa com baixa frequência (católicas, - $1 \mathrm{vz} /$ semana), protestantes que frequentam o culto com alta frequência (protestantes, 1 ou + vzs/semana), protestantes que frequentam o culto com baixa frequência (protestantes, - $1 \mathrm{vz}$ / semana, pentecostais que frequentam o culto com alta frequência (pentecostais, 1 ou + vzs/seman), e pentecostais que frequentam o culto com baixa frequência (pentecostais, $-1 \mathrm{vz} /$ semana).

As seguintes covariáveis foram incluídas nos modelos multivariados: dois grupos etários: 15-19 (grupo de referência) e 20-24 anos, três níveis de escolaridade: 0 a 4 anos de estudo (grupo de referência), 5 a 8 anos, e 9 anos e mais, se reside em área rural ou urbana atualmente (rural é a categoria de referência), onde morou durante a infância (morava em cidade pequena quando tinha 12 anos é a referência), e região de residência (Nordeste é a referência). Este trabalho também avaliou o status socioeconômico (SSE) do domicílio onde a jovem reside, usando um método sugerido pela
Associação Brasileira de Empresa de Pesquisas (ABEP) (texto não publicado). Este método emprega informações sobre educação do chefe do domicílio além de nove variáveis de consumo dentro do domicilio, que incluem: número de televisões, DVDs, rádios, banheiros, carros, máquinas de lavar, geladeiras, freezers, e número de empregadas domésticas. As PNDSs de 1996 e 2006 disponibilizam estas informações para o cálculo do indicador agregado do SSE, que varia entre zero e sete (nível mais elevado de consumo) e é tratado como uma variável contínua.

\section{RESULTADOS}

A Tabela 1 apresenta a distribuição da amostra segundo nossas variáveis explicativas para as jovens entre 15 e 24 anos no Brasil para cada ano analisado. Enquanto 56,6\% das jovens em 1996 tinham entre 15 e 19 anos, em 2006 este percentual caiu para 49,3\%. Em relação à escolaridade, 30,2\% das jovens reportaram ter 9 anos de estudo ou mais em 1996 e, como esperado, devido a expansão educacional, este percentual aumentou substancialmente, chegando a $57,6 \%$ em 2006. As categorias das demais variáveis na Tabela 1 apresentam variações menos expressivas entre 1996 e 2006. Uma exceção é a média do índice socioeconômico, que aumentou 1,8 para 2,1 durante este período de dez anos.

A Tabela 2 mostra que a distribuição das jovens entre as filiações religiosas confirma o declínio do catolicismo e o aumento do protestantismo (de 13,0 para $24,1 \%$ ) no período analisado. Ao mesmo tempo, diminuiu o percentual daquelas que nunca vão à igreja, enquanto ficou praticamente constante o percentual daquelas que atendem cultos ou missas 1 ou + vzs/semana. Além disso, os resultados da Tabela 2 indicam que, nos dois anos de estudo, as protestantes entre 15 e 24 anos frequentam mais assiduamente a igreja do que as católicas. Mais de $70 \%$ das jovens protestantes em 1996 e das protestantes de igrejas tradicionais e pentecostais em 2006 frequentavam cultos $1 \mathrm{ou}+\mathrm{vzs} /$ semana. Este percentual é bem inferior entre as católicas, tanto em 1996 como em 2006.

A idade mediana ao ter o primeiro filho durante a adolescência e antes do casamento foi anos tanto em 1996 como em 2006 (resultados não mostrados). Os resultados dos modelos multivariados da Tabela 3 mostram que filiação religiosa e frequência aos cultos ou missas estão associadas com a idade ao ter o

TABELA 1. Distribuição das variáveis explicativas entre as jovens de 15 a 24 anos no Brasil.a PNDSs de 1996 e 2006

\begin{tabular}{|c|c|c|c|c|}
\hline \multirow[b]{2}{*}{ Variável } & \multicolumn{2}{|c|}{$1996(n=4528)$} & \multicolumn{2}{|c|}{$2006(n=4996)$} \\
\hline & No. & $\%$ & No. & $\%$ \\
\hline \multicolumn{5}{|l|}{ Idade (anos) } \\
\hline $15-19$ & 2537 & 56,6 & 2489 & 49,3 \\
\hline $20-24$ & 1991 & 43,4 & 2507 & 50,7 \\
\hline \multicolumn{5}{|l|}{ Escolaridade } \\
\hline 0 a 4 anos & 1319 & 26,3 & 452 & 8,4 \\
\hline 5 a 8 anos & 1932 & 43,5 & 1772 & 34,0 \\
\hline$\geq 9$ & 1277 & 30,2 & 2772 & 57,6 \\
\hline \multicolumn{5}{|l|}{ Residência atual } \\
\hline Rural & 852 & 18,6 & 1439 & 17,9 \\
\hline Urbano & 3676 & 81,4 & 3557 & 82,1 \\
\hline \multicolumn{5}{|l|}{ Residência na infância } \\
\hline Cidade pequena & 2842 & 60,8 & 3127 & 55,2 \\
\hline Cidade grande/Capital & 1686 & 39,2 & 1869 & 44,8 \\
\hline \multicolumn{5}{|l|}{ Região } \\
\hline Nordeste & 1861 & 30,9 & 1098 & 28,9 \\
\hline Norte & 553 & 5,8 & 937 & 8,6 \\
\hline Sudeste & 1174 & 41,4 & 1012 & 42,3 \\
\hline Sul & 463 & 14,5 & 933 & 13,3 \\
\hline Centro-oeste & 477 & 7,4 & 1016 & 7,0 \\
\hline SSEb (Média) & & 1,8 & & 2,1 \\
\hline
\end{tabular}

Fonte: Pesquisa Nacional de Demografia e Saúde de 1996 e Pesquisa Nacional de Demografia e Saúde de Mulheres e Crianças de 2006.

a As distribuições relativas foram calculadas levando-se em consideração informações do plano amostral e dos pesos da PNDS, tornando assim as estimativas representativas da população e não da amostra.

b Status socioeconômico: varia entre 0 y 7 (nível mais elevado de consumo). 
TABELA 2. Distribuição da filiação religiosa segundo frequência aos cultos ou missas entre mulheres de 15 a 24 anos no Brasil

\begin{tabular}{|c|c|c|c|c|c|c|c|c|c|c|c|c|}
\hline \multirow[b]{3}{*}{ PNDS } & \multirow[b]{3}{*}{ Filiação religiosa } & \multicolumn{11}{|c|}{ Frequência aos cultos ou missas } \\
\hline & & \multicolumn{2}{|c|}{1 ou + vzs/semana } & \multicolumn{2}{|c|}{$-1 \mathrm{vz} /$ semana } & \multicolumn{2}{|c|}{$-1 \mathrm{vz} / \mathrm{mês}$} & \multicolumn{2}{|c|}{ Nunca } & \multicolumn{2}{|c|}{ Total } & \multirow[b]{2}{*}{ Valor de $P$} \\
\hline & & No. & $\%$ & No. & $\%$ & No. & $\%$ & No. & $\%$ & No. & $\%$ & \\
\hline \multirow[t]{5}{*}{1996} & Católica & 1182 & 33,2 & 989 & 26,8 & 424 & 11,7 & 960 & 28,4 & 3562 & 77,7 & $<0,001$ \\
\hline & Protestante & 439 & 76,4 & 71 & 12,2 & 21 & 3,9 & 46 & 7,6 & 577 & 13,0 & \\
\hline & Outras religiões & 58 & 63,3 & 18 & 20,3 & 4 & 2,9 & 15 & 13,6 & 95 & 2,6 & \\
\hline & Sem religião & 0 & 0,0 & 0 & 0,0 & 0 & 0,0 & 294 & 100,0 & 294 & 6,7 & \\
\hline & Total & 1679 & 37,4 & 1078 & 22,9 & 449 & 9,7 & 1315 & 30,1 & 4528 & 100,0 & \\
\hline \multirow[t]{6}{*}{2006} & Católica & 1105 & 30,9 & 675 & 21,4 & 934 & 29,7 & 505 & 18,0 & 3230 & 60,4 & $<0,001$ \\
\hline & Protestante tradicional & 396 & 71,5 & 86 & 10,9 & 83 & 14,4 & 20 & 3,2 & 585 & 13,8 & \\
\hline & Pentecostal & 387 & 77,8 & 52 & 12,9 & 30 & 8,0 & 13 & 1,3 & 483 & 10,3 & \\
\hline & Outras religiões & 78 & 40,2 & 20 & 16,6 & 33 & 20,1 & 42 & 23,1 & 175 & 3,8 & \\
\hline & Sem religião & 32 & 5,1 & 29 & 3,9 & 90 & 20,2 & 368 & 70,8 & 523 & 11,7 & \\
\hline & Total & 1998 & 38,7 & 862 & 16,8 & 1170 & 23,9 & 948 & 20,5 & 4996 & 100,0 & \\
\hline
\end{tabular}

Fonte: Pesquisa Nacional de Demografia e Saúde de 1996 e Pesquisa Nacional de Demografia e Saúde de Mulheres e Crianças de 2006.

primeiro filho, dado que este evento ocorreu durante a adolescência e antes do casamento. O Modelo 1 indica que as jovens que pertenciam a uma igreja protestante em 1996 estavam expostas a um menor risco de ter um filho antes do casamento e antes de completar 20 anos (RR $=0,60 ; P<0,10)$ do que as jovens católicas. Por outro lado, aquelas que reportaram não ter uma filiação religiosa apresentaram um risco $70 \%$ maior de serem mães solteiras e adolescentes $(\mathrm{RR}=1,70 ; P<0,05)$, mesmo quando controlando por variáveis demográficas e socioeconômicas. No mesmo ano, o Modelo 2 mostra que jovens que frequentavam cultos ou missas - $1 \mathrm{vz} /$ semana ou 1 ou + vzs/semana também estavam sob um menor risco de serem mães durante a adolescência e antes do casamento do que aquelas que nunca frequentavam cultos ou missas.

O Modelo 3 mostra que o risco de ter o primeiro filho na adolescência e antes do casamento varia dentro da mesma filiação religiosa, segundo a frequência aos cultos ou missas. Em 1996, católicas que frequentavam a missa 1 ou + vzs / semana estavam substancialmente e significativamente menos expostas a este risco do que aquelas que frequentavam - $1 \mathrm{vz} /$ semana $(\mathrm{RR}=0,64 ; P<0,05)$. $\mathrm{O}$ mesmo ocorreu entre as protestantes que reportaram alta frequência aos cultos, contudo o risco entre elas era ainda menor $(\mathrm{RR}=0,49 ; P<0,05)$.

De acordo com o Modelo 4, jovens protestantes continuam a apresentar um risco de ser mãe solteira na adolescência inferior ao das católicas em 2006 (RR = $0,59 ; P<0,10)$. No entanto, quando estas jovens são analisadas separadamente no Modelo 4.1, de acordo com a igreja protestante que fazem parte (tradicional ou pentecostal), fica claro que as pentecostais apresentam um risco bem inferior de serem mães adolescentes e antes do casamento ( $R R=0,42 ; P<0,05)$ do que as católicas, enquanto as protestantes tradicionais deixam de apresentar um risco estatisticamente significante.

O Modelo 5 indica que uma maior frequência aos cultos ou missas ainda está associado com um menor risco de fecundidade na adolescência e antes do casamento, contudo apenas entre aquelas que vão à igreja 1 ou + vzs/semana o resultado é estatisticamente significativo. Por último, o Modelo 6 mostra que jovens pentecostais, independente da frequência com que iam aos cultos, estavam sob um menor risco de serem mães adolescentes e antes do casamento em 2006 do que católicas que frequentam - $1 \mathrm{vz} /$ semana a igreja.

Como esperado, os resultados das demais variáveis explicativas da Tabela 3 confirmam que escolaridade, região de residência e nível socioeconômico do domicílio são estatisticamente associados com a idade ao ter o primeiro filho na adolescência e antes do casamento.

\section{DISCUSSÃO}

O principal objetivo deste estudo foi examinar a associação entre religião (medida através da filiação religiosa e frequência aos cultos ou missas) e a idade ao ter o primeiro filho no Brasil, dado que este evento ocorreu durante a adolescência e antes do casamento. Os resultados indicam uma forte associação entre fecundidade pré-marital na adolescência e envolvimento religioso, tanto em 1996 como em 2006. No entanto, foi identificada uma inversão na influência das variáveis de religião nos dois anos analisados.

Em 1996, a frequência aos cultos ou missas foi mais importante na explicação da idade ao primeiro filho do que a filiação religiosa. Tanto as jovens católicas como as protestantes que frequentavam missas ou cultos 1 ou + vzs/semana, apresentaram um risco substancialmente e significativamente inferior de serem mães adolescentes e antes do casamento, quando comparadas com as católicas que frequentam missas - $1 \mathrm{vz} /$ semana. Estes resultados reforçam a relevância em se empregar uma medida de religiosidade neste trabalho. No entanto, é importante considerar a associação, mesmo que indireta, entre a idade ao ter o primeiro filho e o pertencimento a uma igreja protestante, já que estas parecem estimular mais ativamente a frequência de seus fiéis à igreja do que a igreja católica.

Em 2006, o pertencimento a uma igreja pentecostal é predominante na explicação da idade ao primeiro filho na adolescência e antes do casamento. Independente da frequência aos cultos, jovens de igrejas pentecostais apresentaram um risco muito inferior de terem filhos na adolescência e antes do casamento do que católicas que frequentam - $1 \mathrm{vz} /$ semana a igreja.

Como mencionado anteriormente, a religião passou a receber, recentemente, maior destaque em estudos sobre o comportamento sexual e reprodutivo de 
TABELA 3. Razões de risco de ter o primeiro filho antes do casamento e durante a adolescência entre mulheres de 15 e 24 anos no Brasil

\begin{tabular}{|c|c|c|c|c|c|c|c|}
\hline \multirow[b]{2}{*}{ Variável } & \multicolumn{3}{|c|}{1996} & \multicolumn{4}{|c|}{2006} \\
\hline & Modelo 1 & Modelo 2 & Modelo 3 & Modelo 4 & Modelo 4.1 & Modelo 5 & Modelo 6 \\
\hline \multicolumn{8}{|l|}{ Idade (anos) } \\
\hline $15-19$ & 1.00 & 1.00 & 1.00 & 1.00 & 1.00 & 1.00 & 1.00 \\
\hline $20-24$ & 1.21 & 1.20 & 1.18 & 1.48 & 1.50 & 1.47 & 1.50 \\
\hline \multicolumn{8}{|l|}{ Escolaridade } \\
\hline 0 a 4 anos & 1.00 & 1.00 & 1.00 & 1.00 & 1.00 & 1.00 & 1.00 \\
\hline 5 a 8 anos & $0.75^{a}$ & 0.80 & 0.78 & 0.61 & 0.63 & 0.67 & 0.64 \\
\hline$\geq 9$ & $0.20^{\mathrm{b}}$ & $0.22^{\mathrm{b}}$ & $0.21^{b}$ & $0.35^{c}$ & $0.36^{c}$ & $0.38^{c}$ & $0.37^{c}$ \\
\hline \multicolumn{8}{|l|}{ Afiliação religiosa } \\
\hline Católica & 1.00 & & & 1.00 & 1.00 & & \\
\hline Protestante & $0.60^{\mathrm{a}}$ & & & $0.59^{a}$ & 0.75 & & \\
\hline Pentecostal & & & & & $0.42^{c}$ & & \\
\hline Outras religiões & $2.18^{a}$ & & & 0.48 & 0.46 & & \\
\hline Sem religião & $1.70^{c}$ & & & 1.56 & 1.55 & & \\
\hline \multicolumn{8}{|l|}{ Frequência aos cultos ou missas } \\
\hline Nunca & & 1.00 & & & & 1.00 & \\
\hline$-1 \mathrm{vz} / \mathrm{mês}$ & & 0.87 & & & & 0.72 & \\
\hline$-1 \mathrm{vz} /$ semana & & $0.47^{\mathrm{b}}$ & & & & 0.83 & \\
\hline 1 ou + vzs/semana & & $0.44^{\mathrm{b}}$ & & & & $0.46^{c}$ & \\
\hline \multicolumn{8}{|c|}{ Afiliação religiosa por frequência aos cultos ou missas } \\
\hline Católicas com baixa frequência & & & 1.00 & & & & 1.00 \\
\hline Católicas com alta frequência & & & $0.64^{c}$ & & & & $0.59^{a}$ \\
\hline Pentecostais com baixa frequência & & & & & & & $0.26^{c}$ \\
\hline Pentecostais com alta frequência & & & & & & & $0.40^{\mathrm{C}}$ \\
\hline Protestantes ${ }^{d}$ com baixa frequência & & & 0.68 & & & & 0.68 \\
\hline Protestantes ${ }^{d}$ com alta frequência & & & $0.49^{c}$ & & & & 0.64 \\
\hline Outras religiões & & & 1.90 & & & & 0.41 \\
\hline Sem religião & & & 1.50 & & & & 1.37 \\
\hline \multicolumn{8}{|l|}{ Residência atual } \\
\hline Rural & 1.00 & 1.00 & 1.00 & 1.00 & 1.00 & 1.00 & 1.00 \\
\hline Urbano & 1.28 & 1.27 & 1.29 & 1.68 & 1.70 & 1.69 & $1.74^{\mathrm{C}}$ \\
\hline \multicolumn{8}{|l|}{ Residência na infância } \\
\hline Cidade pequena & 1.00 & 1.00 & 1.00 & 1.00 & 1.00 & 1.00 & 1.00 \\
\hline Cidade grande/Capital & 0.89 & 0.85 & 0.87 & 0.96 & 0.96 & 0.95 & 0.95 \\
\hline \multicolumn{8}{|l|}{ Região } \\
\hline Nordeste & 1.00 & 1.00 & 1.00 & 1.00 & 1.00 & 1.00 & 1.00 \\
\hline Norte & $2.38^{b}$ & $2.51^{\mathrm{b}}$ & $2.46^{b}$ & $1.71^{\mathrm{c}}$ & $1.73^{c}$ & $1.85^{\mathrm{c}}$ & $1.84^{\mathrm{C}}$ \\
\hline Sudeste & 1.35 & 1.44 & 1.41 & 1.52 & 1.53 & 1.52 & 1.57 \\
\hline Sul & 1.36 & 1.53 & 1.41 & 1.59 & 1.60 & 1.51 & 1.63 \\
\hline Centro-oeste & $1.87^{c}$ & $2.03^{c}$ & $1.93^{c}$ & $1.74^{\mathrm{c}}$ & $1.76^{c}$ & $1.82^{\mathrm{C}}$ & $1.80^{\mathrm{c}}$ \\
\hline \multicolumn{8}{|l|}{ Status socioeconômico } \\
\hline Média & $0.82^{c}$ & $0.81^{\mathrm{c}}$ & $0.82^{c}$ & $0.62^{b}$ & $0.60^{b}$ & $0.62^{b}$ & $0.62^{b}$ \\
\hline-2 log-likelihood & 3393 & 3355 & 3382 & 4500 & 4500 & 4496 & 4495 \\
\hline
\end{tabular}

Fonte: Pesquisa Nacional de Demografia e Saúde de 1996 e da Pesquisa Nacional de Demografia e Saúde de Mulheres e Crianças de 2006.

a $P<0.1$.

b $P<0.01$.

c $P<0.05$.

d Grupo formado por Protestantes e Evangélicos em 1996 e Protestantes tradicionais em 2006.

adolescentes e jovens brasileiros (8-11). McKinnon, Potter e Garrard-Burnett (8), por exemplo, examinaram a relação entre protestantismo e a chance de ter um filho na adolescência entre mulheres de 15 a 17 anos na região metropolitana do Rio de Janeiro. Este estudo mostra que a chance de ser mãe é substancialmente menor entre adolescentes membros da Igreja Batista, de outras protestantes tradicionais, da Assembléia de Deus ou de outras igrejas pentecostais, quando comparadas com as católicas.
Além disso, os resultados encontrados neste artigo oferecem indícios sobre diferenças dentro da mesma religião, segundo frequência aos cultos ou missas. Esta é uma importante contribuição deste artigo. Ao contrário da filiação religiosa, a frequência aos cultos ou missas é uma medida de religiosidade ou envolvimento religioso (12). Adicionalmente, diferente de trabalhos anteriores, foi possível comparar temporalmente, através dos dados da PNDSs de 1996 e 2006, a associação entre religião e idade ao ter o filho na adolescência.

A associação entre religião e o comportamento sexual e reprodutivo também tem sido observada no contexto internacional. Um número expressivo de estudos investiga, por exemplo, fatores associados à alta fecundidade entre as mulçumanas $(20,21)$. Outros trabalhos, como os de Agha, Hutchinson e Kusanthan (22) e Addai (23) encontraram para Zâmbia e Gana, respectivamente, resultados semelhantes ao do presente 
artigo. De acordo com esses dois estudos, mulheres jovens filiadas em igrejas mais conservadoras tendem adiar o início da vida sexual em relação às jovens de igrejas consideradas mais liberais em se tratando de normas sobre comportamento sexual e reprodutivo.

Este artigo possui algumas limitações decorrentes do uso de dados transversais. Os dados das PNDSs, por exemplo, não permitem estabelecer uma relação causal entre as variáveis de interesse. Ou seja, não é possível dizer se é a religião que influencia o comportamento reprodutivo das jovens ou o contrário. Este trabalho sugere que a religião e, mais precisamente as igrejas pentecostais no Brasil, são capazes de inibir a fecundidade de adolescentes antes do casamento. Contudo, jovens que já tinham uma predisposição a esperarem o casamento para iniciar a vida sexual, podem escolher se tornar membros de uma igreja pentecostal, já que podem encontrar neste espaço um ambiente favorável para manter suas preferências em relação ao comportamento sexual. Da mesma forma, se as igrejas pentecostais promovem sanções punitivas relacionadas ao comportamento sexual e reprodutivo de adolescentes e jovens solteiros, aquelas jovens que engravidam antes do casamento podem preferir deixar a igreja que pertencem para evitar certos constrangimentos, o que pode causar também uma concentração nestas igrejas daquelas que nunca tiveram filhos antes do casamento.

A segunda limitação é a falta de informação sobre variáveis que mudam ao longo do tempo, como a educação e o local de residência, e de interesse particular para este trabalho, a religião e a religiosidade. Como as PNDSs fornecem estas informações no momento em que a pesquisa foi conduzida, e não no momento em que a jovem teve o primeiro filho, não é possível afirmar que ao experimentar este evento, ela era pentecostal ou católica, ou que frequentava a igreja uma vez por semana ou por mês. Ela pode, por exemplo, ter mudado de religião ao longo do tempo.

Mesmo com tais limitações, os resultados aqui apresentados são muito instigantes, na medida em que evidenciam que as protestantes, especialmente pentecostais, apresentam um risco reduzido de serem mães adolescentes. Este resultado não era esperado, já que o pentecostalismo predomina nos grupos populacionais menos favorecidos (24), com menor renda, educação e residentes nas áreas urbanas, onde também se concentra a fecundidade de adolescentes no Brasil.

Esta pesquisa enfatizou os potenciais efeitos positivos da religião no comportamento sexual de adolescentes, mais especificamente o menor risco de fecundidade entre adolescentes de igrejas protestantes, especialmente das pentecostais. No entanto, é importante salientar que efeitos negativos da religião também podem ser percebidos, como por exemplo, aqueles decorrentes da proibição de uso de preservativos em religiões que indicam a abstinência como único método contraceptivo.

Finalmente, é possível sugerir que a religião esteja tomando o espaço da família e da escola na comunicação sobre o comportamento sexual e reprodutivo com adolescentes e jovens no Brasil. Embora muitos estudiosos concordem sobre a necessidade de se promover a discussão de questões referentes à sexualidade entre este público, na prática, pais e educadores ainda apresentam dificuldades em abordar este assunto (25). Sendo assim, se algumas religiões ou igrejas conseguem estabelecer regras de comportamento e ainda oferecem espaço de interação social e aprendizado de técnicas e habilidades, pode-se esperar que elas estejam se tornando um dos poucos canais de informação sobre o tema entre a população jovem do país. Esta hipótese deve ser investigada cuidadosamente, já que os ensinamentos religiosos podem estar a favor, mas também algumas vezes contra o que a família e o Estado entendem como benéfico para o adolescente e o jovem no Brasil.

\section{Conclusão}

A religião tem se tornado um fator cada vez mais importante e presente na vida de adolescentes e jovens no Brasil. $\mathrm{Na}$ tentativa de atrair a atenção deste público, destaca-se o papel das igrejas protestantes pentecostais, que têm oferecido recursos que envolvem grande disponibilidade de tempo e espaço para a atuação desta parcela da população no âmbito religioso. Além disso, com o crescimento do pentecostalismo no Brasil nas últimas décadas, valores e normas conservadoras em relação ao comportamento sexual e reprodutivo de adolescentes e jovens solteiros começaram a ser abordadas, de maneira mais corriqueira e explícita, em igrejas pentecostais.

Este estudo mostrou a existência de uma forte associação entre uma alta frequência aos cultos ou missas e adesão ao pentecostalismo e um reduzido risco de ser mãe adolescente e antes do casamento. Futuros estudos, especialmente de natureza qualitativa, devem ser conduzidos a fim de compreender como os distintos mecanismos da influência religiosa atuam na vida e comportamento de adolescentes no Brasil.

Agradecimentos. À Coordenação de Aperfeiçoamento de Pessoal de Nível Superior (Capes) pelo apoio financeiro dado através do Programa de Apoio a Projetos Institucionais, com a Participação de Recém-doutores (PRODOC) do edital MEC/Capes 029/2010.

\section{REFERÊNCIAS}

1. Gupta N, Mahy M. Sexual initiation among adolescent girls and boys: trends and differentials in Sub-Saharan Africa. Arch Sex Behav. 2003;32(1):41-53

2. Ali MM, Cleland J. Sexual and reproductive behaviour among single women aged 15-24 in eight Latin American countries: a comparative analysis. Soc Sci Med. 2005;60 (6):1175-85.
3. Sociedade Civil Bem-Estar Familiar no Brasil BENFAM. Pesquisa nacional sobre demografia e saúde, 1996. Rio de Janeiro: Sociedade Civil Bem-Estar Familiar no Brasil/ Demographic and Health Survey; 1997.

4. Ministério da Saúde (Brasil). Pesquisa Nacional de Demografia e Saúde da Criança e da Mulher (PNDS-2006). Brasília: MS/ São Paulo: CEBRAP; 2008.
5. Leite IC, Rodrigues RN, Fonseca MC. Fatores associados com o comportamento sexual e reprodutivo entre adolescentes das regiões Sudeste e Nordeste do Brasil. Cad Saude Publica. 2004;20(2):474-81.

6. Duarte CM, Nascimento VB, Akerman M. Adolescent pregnancy and social exclusion: analysis of intra-urban disparities [Gravidez na adolescência e exclusão social: análise 
de disparidades intra-urbanas]. Rev Panam Salud Publica. 2006;19(4):236-43.

7. Marinho LFB, Aquino EM L, Almeida MCC. Contraceptive practices and sexual initiation among young people in three Brazilian State capitals [Práticas contraceptivas e iniciação sexual entre jovens de três capitais brasileiras]. Cad. Saude Publica. 2009;25(2):227-39.

8. McKinnon S, Potter JE, Garrard-Burnett V. Adolescent fertility and religion in Rio de Janeiro, Brazil in the year 2000: the role of Protestantism. Popul Stud (Camb). 2008;62(3): 289-303.

9. Silva CG, Santos AO, Licciardi, DC, Paiva V. Religiosidade, juventude e sexualidade: entre a autonomia e a rigidez. Psicol estud[online]. 2008;13(4):683-92. Disponível em: http:// www.scielo.br/scielo.php? pid=S141373722008000400006\&script=sci_abstract\& tlng=pt

10. Heilborn ML, Peixoto C, Duarte LFD, Barros ML, organizadores. Sexualidade, Família, e Ethos Religioso. Primeira edição. Rio de Janeiro: Garamond; 2005.

11. Paiva V, Calazans G, Venturi G, Dias R. Age and condom use at first sexual intercourse of Brazilian adolescents [Idade e uso de preservativo na iniciação sexual de adolescentes brasileiros]. Rev Saude Publica. 2008;42Suppl 1:45-53.
12. Regnerus MD. Forbidden fruit-Sex and religion in the lives of American teenagers. New York: Oxford University Press; 2007.

13. Smith $\mathrm{C}$. Theorizing religious effects among American adolescents. J Sci Stud Relig. 2003; 42(1):17-30.

14. Burdick J. Looking for God in Brazil. Berkeley: University of California Press; 1993.

15. Mariz CL. Coping with poverty: Pentecostal and Christian Base Communities in Brazil Philadelphia, PA: Temple University Press; 1994.

16. Verona APA. Sexual Initiation and religion in Brazil [Tese de Doutorado em Sociologia] Austin (TX): University of Texas at Austin; 2010.

17. Cavenaghi S. Aspectos metodológicos e comparabilidade com pesquisas anteriores. In: Berquó E, Garcia S, Lago T, organizadores. Dimensões do processo reprodutivo e da saúde da criança. Pesquisa Nacional de Demografia e Saúde da Mulher e da Criança — PNDS 2006. São Paulo: CEBRAP, Brasília: Ministério da Saúde, 2009.

18. Gupta N. Sexual initiation and contraceptive use among adolescent women in northeast Brazil. Stud Fam Plann. 2000;31(3):228-38.

19. Allison PD. Survival analysis using SAS: A practical guide. 1st ed. Cary, NC: SAS; 1995.

20. Morgan SP, Stash S, Smith HL, Mason KO. Muslim and Non-Muslim Differences in
Female Autonomy and Fertility: Evidence from four Asian Countries. Popul Dev Rev. 2002;28:515-37.

21. Westoff CF, Frejka T. Religiousness and Fertility among European Muslims Popul Dev Rev. 2007;33(4):785-809

22. Agha S, Hutchinson P, Kusanthan T. The effects of religious on sexual initiation and condom use in Zambia. J Adolesc Health. 2006; 38(5):550-5

23. Addai I. Religious affiliation and sexual initiation among Ghanaian women. Rev Relig Res. 2000;41(3):328-43.

24. Chesnut RA. Born again in Brazil-the Pentecostal boom and the pathogens of poverty. 1st ed. New Brunswick, NJ: Rutgers University Press. 1997.

25. Alencar RA, Silva L, Silva FA, Diniz RES. Development of a proposal for sex education for adolescents [Desenvolvimento de uma proposta de educação sexual para adolescentes]. Ciênc educ (Bauru) [online]. 2008;14(1):15968. Disponível em: http://www.scielo.br/ pdf/ciedu/v14n1/11.pdf

Manuscrito recebido em 5 de janeiro de 2011. Aceito em versão revisada em 14 de junho de 2011.

ABSTRACT Objective. The objective of this study was to examine the association between the age of having one's first child in adolescence and before marriage and religious involvement in Brazil, measured by religious affiliation and frequency of attendance at religious services or masses.

\section{Religion and fertility among adolescents in Brazil}

Methods. Transverse data obtained from the National Survey of Demographics and Health of 1996 and the National Survey of Demographics and Health of Women and Children of 2006 were utilized. Cox proportional risks models were employed to estimate the association between religion and age of having one's first child premaritally and during adolescence.

Results. The results indicate a strong association between premarital fertility in adolescence and religious involvement in both 1996 and 2006. In 1996, frequency of attendance at religious service $s$ or masses was more important than religious affiliation in explaining the age at which one had her first child. In 2006, belonging to a Pentecostal church comes to predominate.

Conclusions. The results presented in this study are encouraging insofar as they show that Protestant adolescents, particularly Pentecostals, have a reduced risk of adolescent premarital motherhood. This result was not expected, given that Pentecostalism predominates in the less advantaged population groups, with lower incomes and levels of education and residence in urban areas, where adolescent fertility is also concentrated in Brazil. Future studies must be undertaken with the purpose of understanding how the various mechanisms of religious influence operate in the life and behavior of adolescents in Brazil.

Key words Religion and sex; adolescent; sexual behavior; reproductive behavior; Brazil. 